American Journal of Infectious Diseases 7 (3): 55-60, 2011

ISSN 1553-6203

(C) 2011 Science Publications

\title{
Genotoxic Profile of Motor Garage Workers
}

\author{
Nandan M. Shastri and Hemlata Pant \\ Department of Biochemistry, P D Patel Instuitute of Applied Sciences, \\ Charotar University of Science and Technology, Changa, Gujarat, India
}

\begin{abstract}
Problem statement: One way to study the effects on an exposed population is to conduct monitoring studies, using relevant biological parameters with a short term manifestation, such as cytogenetic analysis express the damages to the DNA or to the chromosomes resulting from exposure can be identified. The present study was thus designed to elucidate the effect motor garage environment on the workers. Approach: Peripheral blood culture was performed with blood collected from workers of Motor garages in Ahmadabad, situated at central Gujarat region. Our objective was to establish the relationship of the environment induced Micro Nucleus (MN) and Chromosomal Aberrations (CA) with non-occupational factors, such as the smoking and drinking habits since quality of life and nutritional status are the key factors that influence toxicity profile. Results: On comparing the smokers of the exposed group with that of smokers of control group the structural chromosomal aberration and micronucleus induction frequency was significant high $(\mathrm{p}<0.02)$ in the exposed group. Exposed individual with non smoking habit also exhibited remarkable increase in the CA and MN values with a significant increase $(\mathrm{p}<0.02)$ when compared with control. Considering the drinking habit of the exposed individual to that of the control, the exposed group showed significant increase $(\mathrm{p}<0.05)$ in the CA as well as MN. Conclusion: This indicates that occupational exposure to the many chemicals of the garage environment can have Genotoxic effects and these effects increases with the increase in period of exposure.
\end{abstract}

Key words: Micronucleus induction, chromosomal aberrations, peripheral blood culture, genotoxic effects, exposed group, Micro Nucleus (MN), motor garage

\section{INTRODUCTION}

Knowledge of human health risks related to environmental exposure to hazardous chemical agents is a current concern (Franco et al., 2008; Akan et al., 2009). Populations of industrial areas are intensely exposed to chemical substances that can cause mutations, cancer and congenital defects (Hirvonen, 1995). The majority of situations involve exposures to low doses for long periods, which in many cases involve the individual's entire lifetime and result in small increments in health risks (Franco et al., 2008). Occupational agents can induce several types of cancer, such as urinary tract, skin, larynx and pancreas cancers and leukemia (Santos-Mello and Silva, 1996). One way to study the effects on an exposed population is to conduct monitoring studies, using relevant biological parameters with a short term manifestation, such as cytogenetic analysis, by which damages to the DNA or to the chromosomes resulting from exposure can be identified (Pant and Rao, 2010). The obtained information can be used as an early warning about the potential risk of health problems developing in the long run.

Exposure to a wide range of industrial chemicals including heavy metals, contained in brake fluids, degreasers, detergents, lubricants, metal cleaners, paints, fuel, solvents, resulting in various forms of chronic poisoning.

Air quality measurements in vehicle garages indicate that high pollutant concentrations caused by car-induced emissions may occur in the motor garage (Karahalil et al., 1998). Benzene dusts, $\mathrm{NO}_{2}, \mathrm{PAH}$ and $\mathrm{CO}$ are the main pollutants present in the motor garage. Most garages generate hazardous waste, waste water, air emissions and pollutants from such services as fluid replacement or operations like washing or painting parts. There are several types of solid waste. Lead acid is present in the car batteries which is big pollutant. Along with that the paints which contains many aromatic hydrocarbon also contributes in the pollutant list (Martino-Roth et al., 2002).

Corresponding Author: Hemlata Pant, Department of Biochemistry, P D Patel Institute of Applied Sciences,

Charotar University of Science and Technology, Changa, Gujarat, India

Tel: +919824941305 Fax: +9102697247100 
To augment the quality of the life of the intimate workers and workers related to such small scale industry we carried out this pilot based study. Our objective was to evaluate by MNT and CA the level of damage and to establish the relationship of the Micro Nucleus (MN) frequency and (CA) with non-occupational factors, such as the smoking and drinking habits.

\section{MATERIALS AND METHODS}

Subjects: The present investigation was divided into two groups; test and control. Test group included 5 individuals working in an automotive workshop situated at Narol area, Ahmedabad, Gujarat. Control group consisted 5 age matched individuals not exposed to automotive pollutants, mainly office workers. The health and occupational histories of the subjects and controls with respect to age, sex, duration of exposure, socioeconomic condition, nutritional status, smoking and drinking habits were collected. None of the individual had been exposed to x-rays or had taken antibiotics for three months prior to sampling. The worker do all kind of jobs including spray painting, carving, engine fitting. Many a times without using any protective device. These workers were engaged in work for more than 8 hrs per day with a minimum $5 \mathrm{yrs}$ of exposure duration. Workshop owner and worker were educated about objective of the study and duly signed consent forms were obtained from all workers.

Blood collection: Peripheral blood of 5 individuals within the age group 20-30 yrs was collected in plain screw capped tube coated with heparin. The blood was taken to the laboratory within $2 \mathrm{hrs}$ of collection. Short term lymphocyte cultures were established from heparinised blood according to the method by Hungerford (1965).

Experimental groups: There were two groups formed test (from motor garage) and controls (normal healthy individuals). All these groups were then further divided into subgroups on the basis of smoking and drinking habits.

Preparation of culture media: RPMI 1640 powder was obtained from Himedia Pvt Ltd, Mumbai, was dissolved in $800 \mathrm{~mL}$ autoclaved $\mathrm{D} / \mathrm{W}$. To this $2 \mathrm{gms}$ $\mathrm{NaHCO} 3,100 \mathrm{mg}$ penicillin (100,000 units) and 100 $\mathrm{mg}$ streptomycin were added and dissolved. Then the $\mathrm{pH}$ of solution was adjusted to 7.3. Then the media was filtered by negative pressure vacuum filteration followed by packing and labeling of the media bottles. The procedures from vacuum filtration onwards were carried out in laminar air flow. Prepared media was stored at $4 \mathrm{C}$ after labeling.

Peripheral Blood Lymphocyte Culture (PBLC): Peripheral blood lymphocyte culture was carried out by the standard procedure by Hungerford (1965), with slight modification. Blood was collected in heparinized syringe and was properly mixed to avoid any clot formations. $7 \mathrm{~mL}$ RPM-I1640 media containing 10\% FCS, Penicillin and Streptomycin was used to set up the culture in duplicates. $0.1 \mathrm{~mL}$ of Phytohaemagluttinin (PHA) $\left(1 \mathrm{mg} \mathrm{mL}^{-1}\right)$ and 9 drops of blood were added to each tube, mixed properly and kept in incubator at $37 \mathrm{C}$ after closing it tightly. This day was counted as day zero. At 69 th hr the $20 \mu \mathrm{L}$ colchicine $\left(1 \mathrm{mg} \mathrm{mL}^{-1}\right)$ was added to all the tubes, so as to arrest the cells at metaphase.

Harvesting: After $30 \mathrm{~min}$ incubation with colchicines, the cultures were taken out of the incubator and cells were pelleted down by centrifuging at $2000 \mathrm{rpm}$ for 12 minutes. Then $5 \mathrm{~mL}$ prewarmed hypotonic solution $(0.56 \%)$ was added to the pellet and flushed gently with dropper. The tubes were then incubated at $37^{\circ} \mathrm{C}$ in water bath for $20 \mathrm{~min}$ and then $2 \mathrm{~mL}$ chilled fixative (1:3 acetomethanol) was added, flushed properly and centrifuged at 2000r pm for $20 \mathrm{~min}$. The pellet was washed twice with fixative and finally the pellet was suspended in $0.5 \mathrm{~mL}$ of chilled freshly prepared fixative.

Preparation of slides: Frosted slides were first soaked in chromic acid overnight and then kept under running tap water in horizontal coupling jar. After washing the slides with $\mathrm{D} / \mathrm{W}$, they were then transferred to a beaker filled with chilled D/W. then 34 drops of suspension was added to the slides and flamed on the spirit lamp. Slides were then observed under the microscope after air drying.

Giemsa staining: Slides were dipped in $2 \%$ Giemsa for $3 \mathrm{~min}$ and then carefully rinsed with tap water in coupling jar. After air drying, slides were observed under the microscope for well spread metaphase plates to screen for chromosomal aberrations including structural aberrations and numerical aberration (viz chromosomal gaps, breaks, aneuploidy).

Structural aberrations analysis: Giemsa stained metaphase plates were scored for the chromosomal aberrations. The aberrations can be divided into two groups chromosomal and chromatids aberrations. Chromosome aberrations are one where both chromatids are affected at the same loci while in 
chromatids or mis alignment of proximal and distal parts of chromatids are observed with unstained region then it was scored for gaps/breaks. Chromatids gaps includes unstained region not larger than the diameter of chromatids and breaks includes unstained region more than the diameter of chromatid. Similiary chromosome aberrations were also divided into two types, based on the same criteria.

Binucleate induction and Micro Nucleus (MN) analysis: Binucleate induction and micronucleus analysis was done following the method of Fenech (2000) with slight modifications. Cytokinesis was inhibited by the addition of cytochalasin $\mathrm{B}$ to the cultures. With this technique it is possible to observe the products of single cell division and to assess the rate of micronucleus formation in the cell division. Whole blood cultures were established by standard procedure as described earlier. After $72 \mathrm{hrs}$ of incubation, cytochalisine B was added to a final concentration of $30 \mathrm{n} \mu \mathrm{L} / \mathrm{mL}$. At $96 \mathrm{hrs}$ the culture were centrifuged and 5 $\mathrm{mL}$ pre-warmed $\left(37^{\circ} \mathrm{C}\right) 0.075 \mathrm{M} \mathrm{KCl}$ was added to the tube and kept for $25 \mathrm{~min}$ at $37^{\circ} \mathrm{C}$. The cells were then fixed in fresh chilled fixative and kept in ice bath for 20 $\min$. The tubes were centrifuged and cells were given one wash with fixative. The total period of fixative was about $2 \mathrm{~h}$. The slides were stained in $4 \%$ giemsa. Binucleates were scanned for micronucleus under high power objective for each individual at least 500 binucleates were scanned.

Nuclear Division Index (NDI): NDI is often calculated according to the method of (Eastmond and Tucker, 1989). Five hundred viable cells are scored to determine the frequency of cells with 1,2, 3 or 4 nuclei and calculate the NDI using the formula:

$\mathrm{NDI}=\mathrm{M} 1+2(\mathrm{M} 2)+3(\mathrm{M} 3)+4(\mathrm{M} 4) / \mathrm{N}$

where, 1-M4 represent the number of cells with one to four nuclei and $\mathrm{N}$ is the total number of viable cells scored. The NDI and the proportion of binucleated cells are useful parameters for comparing the mitogenic response of lymphocytes and cytostatic effects of agents examined in the assay.

\section{RESULTS}

Mitotic index: The result obtained for the mitotic index and the structural aberration studies between the exposed motor garage workers and normal non exposed individual control is given in the table below (Fig. 1). The mean MI in exposed individual was $6.02+0.6265$ which was slight higher when compared to control individual which showed a MI of $3.70+0.254$. The value of mitotic index for the other group did not showed any significant increase when compared to that of control.

The Mitotic Index of exposed individual is elevated in comparison to controls. The MI is seen high in the workers having the drinking and smoking habit.

Chromosomal aberrations: Increase in the frequency of structural aberration of exposed individual when compared with the control. The mean SCA in the exposed individual was $13.16+5.928$ while in the non exposed control it was $3.0+1.643$. On comparing the smokers of the exposed group with that of smokers of control group the structural chromosomal aberration was significant high $(\mathrm{p}<0.02)$ in the exposed group. Exposed individual with non smoking habit also exhibited remarkable increase in the SCA value with a significant increase $(p<0.02)$ when compared with control. Considering the drinking habit of the exposed individual to that of the control, the exposed group showed significant increase $(\mathrm{p}<0.05)$ in the SCA. There was increase in the SCA value for the non drinking exposed group when compared to control but there was not statistically significant (Fig. 2).

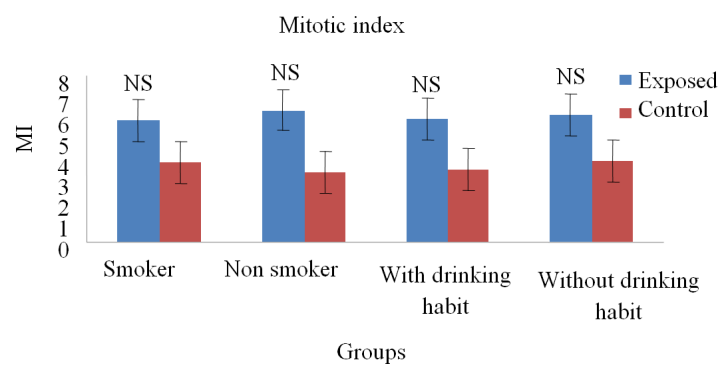

Fig. 1: The mitotic index of the exposed individual compared to controls, Non Significant (NS)

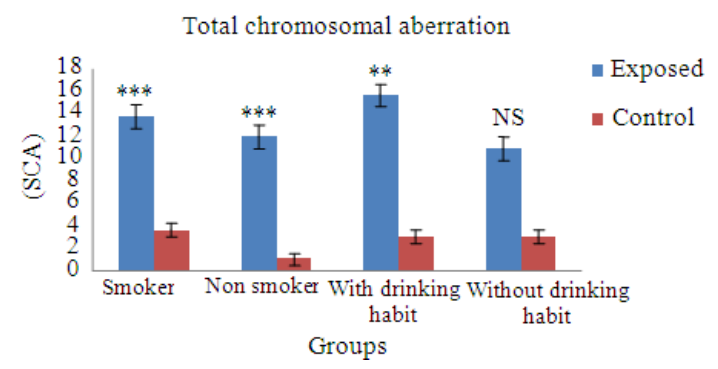

Fig. 2: Increase in the total chromosomal aberration is seen in the exposed group in comparison to non exposed control group, $* * *$ Significant at $\mathrm{p}<0.02$, ** significant at $\mathrm{p}<0.05$, Non Significant (NS) when compared to respective control 


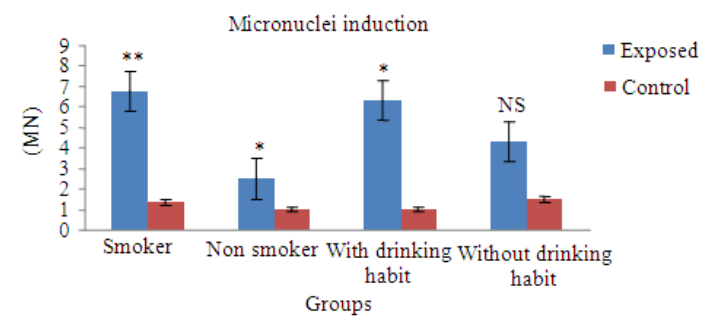

Fig. 3: Micronuclei induction of individual exposed to pollutants in motor garage compared to normal control individuals $* *$ Significant $\mathrm{p}<0.05$, *significant $\mathrm{p}<0.01$, Non Significant (NS)

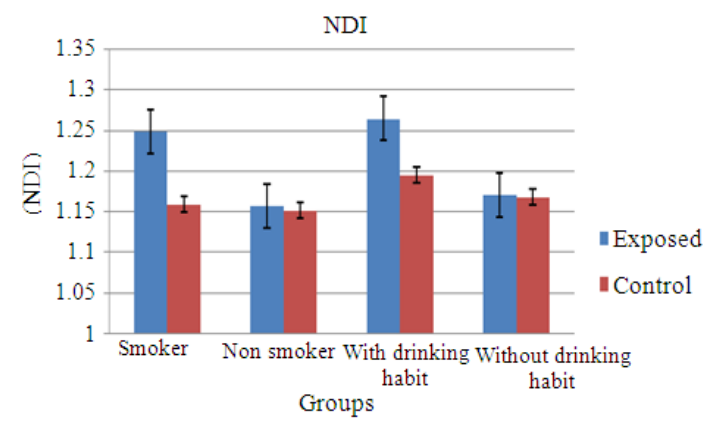

Fig. 4: Exposed individual show increase in the NDI compared to non exposed control, Non Significant (NS)

Micronuclei: The results obtained for the stimulation of micronuclei in individual with exposure to motor garage pollution in comparison to normal control individual is in Fig 3. A remarkable increase $(\mathrm{p}<0.01)$ in the frequency of $\mathrm{MN}$ induction was observed in comparison to the control group. On comparing the smokers of the exposed group with that of smokers of control group the induction of MN was significantly high $(\mathrm{p}<0.05)$ in exposed group. Exposed individuals with no smoking habits also exhibited a remarkable increase $(\mathrm{p}<0.01)$ in the $\mathrm{MN}$ frequency compared to non smoker controls. Considering the drinking habits, exposed group showed a significant rise $(\mathrm{p}<0.01)$ in the induction $\mathrm{MN}$ to control group with drinking habit. However comparing the exposed and control group, without drinking habit, did not show any significant rise in the frequency of MN induction.

It is seen from the graph that the frequency of $\mathrm{MN}$ formation is higher in exposed workers compared to controls. Smoking and drinking habit also shows some correlation with the frequency of MN formation.

NDI: The result obtained from the NDI of the present study doesn't show any statistically significant increased in the exposed individual when compared to the non exposed control (Fig 4). The NDI for the smoking habit individual showed increased in NDI index when compared to their control, same was the observation in the case of the individual wit drinking habit. But there was no significant increase seen in the non smoker of the exposed group when compared with control. The exposed individual also showed the same result as shown by the non smoker group.

\section{DISCUSSION}

This result is in accord with the findings of Santos-Mello and Silva (1996), who investigated chromosome alterations in car painters from the city of Brasilia, discovering a significant increase in chromosome and/or Chromatid anomalies (96\%) in the risk group, as compared to the control group $(55 \%)$. They found a deletion rate of $60 \%$ in the painters and of $20 \%$ in the controls, a proportion similar to the one found in this study.

Car painters are exposed to the action of a great number of chemicals, such as solvents whose foundation is ketone, aliphatic and aromatic compounds and esters, organic and inorganic pigments and several types of resins whose basis is urethane, isocyanate, besides thinner, which contains benzene. Santos-Mello and Cavalcante (1992) performed a cytogenetic evaluation in gas station workers in the cities of São Paulo and Rio de Janeiro and found a significant increase in the frequency of chromosome deletions in the metaphases of the exposed individuals $(0.829 \%)$, as compared to controls $(0.126 \%)$.

Among mechanics, the custom of using petrol to clean their hands during car or engine repair is incessant; therefore, both mechanics and gas station workers inhale petrol gases which contain Genotoxic substances, such as benzene and the products derived from engine ignition. These products, when they enter the blood circulation, also present cytotoxic and genotoxic properties, as demonstrated by Hadnagy and Seemayer (1988).

Karahalil et al. (1998) evaluated workers of oil and petrol engine repair garages, using the Sister Chromatid Exchange (SCE) test and the presence of micronuclei in peripheral blood lymphocytes. The exposed group presented an average of $1.87 \mathrm{MN}$ and the control group an average of $1.56 \mathrm{MN}$, which is a significant difference at the $p<0.05$ level. The results of both tests were in agreement with each other. They also agree with the data obtained in this study.

The smoking habit is widely accepted as a major cause of bladder cancer, since $50 \%$ of the cases were 
identified in smoking men and $1 / 3$ in smoking women. Burgaz et al. (1995) found a significant increase in micronucleated cells $(\mathrm{p}<0.001)$ in smokers, as compared to non-smokers. This is confirmed by the data obtained by us.

Alcoholic beverages have been described as containing mutagenic substances (Maluf and Erdtmann, 2000). Bishop et al. (1997) mention that alcohol does not induce mutations in mammal cells in vitro, whereas in vivo it induces a variety of genetic effects, including sister chromatid exchange and the production of micronuclei, where the evidence, however, is limited to certain test systems or tested organisms. According to Dittberner et al. (1997), alcohol use can increase the number of micronuclei. In the present work there is increase in the frequency of induction in the $\mathrm{MN}$ in the exposed individual consuming alcohol.

Stich and Rosin (1984) evaluated smoking and alcohol consumption over $\mathrm{MN}$ frequency, in order to detect the synergistic effect of these habits. They verified that smokers who drank alcohol presented a significantly increased frequency of $\mathrm{MN}$ in relation to the other interactions.

In cell cultures of peripheral lymphocytes, cytogenetic alterations like chromosomal aberrations, sister chromatid exchange and micronucleus induction have been applied as biomarkers of exposure and early effect in exposures to genotoxic carcinogens (Norppa, 2004; Albertini, 2000). The relevance of increased frequency of cytogenetic alterations as biomarkers of cancer risk has been corroborated by epidemiological studies suggesting the high frequency of chromosomal aberrations as the best predictive parameter for increased cancer risk (Franco et al., 2008).

Young workers in engine repair workshops are exposed to PAHs from engine exhaust and used engine oil. CA frequencies were found to be higher for exposed subjects $(\mathrm{p}<0.05)$. The levels of $1-\mathrm{OH}-\mathrm{pyrene}$ were higher not only in the exposed group compared with controls $(\mathrm{p}<0.001)$ but also in exposed nonsmokers compared with exposed smokers $(\mathrm{p}<0.05)$ (Karahalil et al., 1998). Smith et al. (1998) used painting probes for chromosomes 8 and 21. They observed an increase in the hyperdiploidy of chromosomes 8 and 21 and translocations between chromosomes 8 and 21 with exposure to benzene concentrations higher than $380 \mathrm{mg} \mathrm{m}^{-3}$ Zhang et al. (1998) used FISH in the same group to determine specific aberrations in chromosomes 1, 5 and7. Exposure to benzene was associated with increases in the rates of monosomy 5 and 7 and with increases inthe trisomy and tetrasomy frequencies of all three chromosomes. This result demonstrates that the leukemia specific changes in chromosomes 5 and 7 can be detected by FISH in the peripheral blood of healthy exposed workers.

Several organs are believed to be susceptible to tumor formation after exposure to PAHs. These include the lungs (in particular the bronchi), the skin, the esophagus and colon, the pancreas, the bladder and the breast in women.

In corroboration to these results our study supports the findings that the environ ment in motor garages is genotoxic to human and smoking habit increases the genotoxic effects of motor garage pollutants.

\section{CONCLUSION}

Genotoxic studies are foremost for any occupational exposure studies. Evaluation based on genotoxic parameters is often useful in warranting environmental endowment and occupational health.

The results of this study have shown increased chromosomal aberration as well as increased frequency of cells with micronuclei in motor garage workers as compared to non exposed controls. This indicates that occupational exposure to the many chemicals of the garage environment can have genotoxic effects and these effects increases with the increase in period of exposure.

\section{REFERENCES}

Akan, J.C., F.I.A. Abdulrahman, V.O. Ogugbuaja and J.T. Ayodele, 2009. Heavy metals and anion levels in some samples of vegetable grown within the vicinity of Challawa industrial area, Kano State, Nigeria. Am. J. Applied Sci., 6: 534-542. DOI: 10.3844/ajassp.2009.534.542

Albertini, R.J., D. Anderson, G.R. Douglas, L. Hagmar and K. Heminiki et al., 2000. IPCS guidelines for the monitoring of genotoxic effects of carcinogens in humans. Mut. Res., 463: 111-172. DOI: 10.1016/S1383-5742(00)00049-1

Bishop, J.B., K.L. Witt and R.A. Sloane, 1997. Genetic toxicities of human teratogens. Mut. Res., 396: 943. DOI: 10.1016/S0027-5107(97)00173-5

Burgaz, S., A. Iscan, Z.K. Büyükbingöl, A. Bozkurt and A.E. Karakaya, 1995. Evaluation of micronuclei in exfoliated urothelial cells and urinary thioether excretion of smokers. Mut. Res., 335: 163-169. PMID: 7477047

Dittberner, U., B. Schmetzer, P. Golzer, G. Eisenbrand and H. Zankl, 1997. Genotoxic effects of 2-transhexenal in human buccal mucosa cells in vivo. Mut. Res., 390: 161-165. PMID: 9150764 
Eastmond, D.A. and J.D. Tucker, 1989. Identification of aneuploidy-inducing agents using cytokinesisblocked human lymphocytes and an antikinetochore antibody. Environ. Mol. Mutagen., 13: 34-43. DOI: 10.1002/em.2850130104 PMID: 2783409

Fenech, M., 2000. The in vitro micronucleus technique. Mut. Res., 455: 81-95. DOI: 10.1016/S00275107(00)00065-8

Franco, S.S., A.C. Nardocci and W.M. Gunther, 2008. PAH biomarkers for human health risk assessment: A review of the state-of-the-art. Cad. Saude Publica, 24: 569-580. PMID: 18797723

Hadnagy, W. and N.H. Seemayer, 1988. Cytotoxic and genotoxic effects of extract of particulate emission from a gasoline-powered engine. Environ. Mol. Mutagen, 12: 385-396. PMID: 2461301

Hirvonen, A., 1995. Genetic factors in individual responses to environmental exposures. J. Occup. Environ. Med., 37: 37-43. DOI: 10.1097/00043764-199501000-00006 PMID: 7620941

Hungerford, D.A., 1965. Leukocytes cultured from small inocula of whole blood and the preparation of metaphase chromosomes by treatment with hypotonic KCl. Stain Technol., 40: 333-338. PMID: 5866557

Karahalil, B., S. Burgaz, G. Fisek and A.E. Karakaya, 1998. Biological monitoring of young workers exposed to polycyclic aromatic hydrocarbons in engine repair workshops. Mut. Res., 412: 261-269. PMID: 9600694

Maluf, S.W. and B. Erdtmann, 2000. Evaluation of occupational genotoxic risk in a Brazilian hospital. Genet. Mol. Biol., 23: 485-488. DOI: $10.1590 /$ S $1415-47572000000200040$
Martino-Roth, M.G., J. Viegas, M. Amaral, L. Oliveira and F.L.S. Ferreira et al., 2002. Evaluation of genotoxicity through micronuclei test in workers of car and battery repair garages. Genet. Mol. Biol., 25: 495-500. DOI: $10.1590 / \mathrm{S} 1415-$ 47572002000400021

Norppa, H., 2004. Cytogenetic biomarkers and genetic polymorphisms. Toxicol. Lett., 149: 309-334. DOI: 10.1016/j.toxlet.2003.12.042 PMID: 15093278

Pant, H.H. and M.V. Rao, 2010. Evaluation of in vitro anti-genotoxic potential of melatonin against arsenic and fluoride in human blood cultures. Ecotoxicol. Environ. Safety, 73: 1333-1337. DOI: 10.1016/j.ecoenv.2010.05.004 PMID: 20646762

Santos-Mello, R. and B. Cavalcante, 1992. Cytogenetic studies on gas station attendants. Mut. Res., 280: 285-290. DOI: 10.1016/0165-1218(92)90059-9

Santos-Mello, R. and J.M.G.C. Silva, 1996. Chromosomal aberrations in lymphocytes from car painters. Mut. Res., 368: 21-25. DOI: 10.1016/S0165-1218(96)90036-1

Smith, M.T., L. Zhang, Y. Wang, R.B. Hayes and G. Li et al., 1998. Increased translocations and aneusomy in chromosomes 8 and 21 among workers exposed to benzene. Cancer Res., 58: 2176-2181. PMID: 9605763

Stich, H.F. and M.P. Rosin, 1984. Micronuclei in exfoliated human cells as a tool for studies in cancer risk and cancer intervention. Can. Lett., 22: 241-253. DOI: 10.1016/0304-3835(84)90159-9

Zhang, L., N. Rothman, Y. Wang, R.B. Hayes and G. $\mathrm{Li}$ et al., 1998. Increased aneusomy and long arm deletion of chromosomes 5 and 7 in the lymphocytes of Chinese workers exposed to benzene. Carcinogenesis, 19: 1955-1961. DOI: 10.1093/carcin/19.11.1955 PMID: 9855009 